hep-th/0309170

SCIPP-2000/04

\title{
Is There A String Theory Landscape?
}

\author{
T. Banks, M. Dine and E. Gorbatov \\ Santa Cruz Institute for Particle Physics, Santa Cruz CA 95064
}

\begin{abstract}
We examine recent claims of a large set of flux compactification solutions of string theory. We conclude that the arguments for AdS solutions are plausible. The analysis of meta-stable dS solutions inevitably leads to situations where long distance effective field theory breaks down. We then examine whether these solutions are likely to lead to a description of the real world. We conclude that one must invoke a version of the anthropic principle which insists on carbon based life forms. We explain why it is likely that this leads to a prediction of low energy supersymmetry breaking, but that many features of anthropically selected flux compactifications are likely to disagree with experiment.
\end{abstract}




\section{Introduction}

String Theory has always been plagued by a plethora of solutions which do not describe the real world. Early hopes that some of the highly supersymmetric (SUSic) moduli spaces of solutions would prove inconsistent once nonperturbative physics was understood, were dashed in a definitive manner by the second superstring revolution. More recently there have been claims $[1,2,3,4,5,6,7]$ that there is a large set of additional solutions of string theory, both SUSic and SUSY violating, with a discretuum of values of the cosmological constant. Susskind[8] has dubbed this discrete set of solutions the landscape of string theory, and suggested that anthropic arguments will have to be invoked to explain why the world we see is chosen from among this vast class of solutions. Douglas[9], has carried through what might be described as a prototype counting of such vacua. Ultimately, one might hope determine the distribution of couplings among the states of the landscape (what Douglas refers to as the "ensemble").

The purpose of this paper is to assess the arguments for the existence of the flux discretuum, as well as the prospects that any of these solutions could describe experimental physics. Our conclusions may be summarized as follows: the arguments for the existence of AdS solutions, both SUSic and SUSY violating, appear plausible. The key to verifying their existence would be the discovery of dual CFT's for each of these solutions. Silverstein[10] has suggested a method for constructing these duals using brane/flux transitions. The meta-stable dS solutions are on much less firm ground. Many, perhaps all, of them are likely to tunnel to Big Crunch cosmologies, where the low energy effective field theory technology, which is the primary tool for constructing these solutions, breaks down. We argue that these instabilities must be faced even if the amplitudes for them are much smaller than those for other decay modes. Furthermore, even in the absence of these instabilities, the putative stable state into which these solutions decay, is a Big Bang universe where effective field theory breaks down. Unless we develop techniques for understanding these singularities, there is no reliable evidence that these solutions are approximations to meaningful models of quantum gravity.

The existence of a discretuum of stable or metastable states would force us to adopt a new paradigm for scientific explanation ${ }^{1}$. We will discuss the possible paradigms, but, as we will explain, at least within the classes of flux vacua which have been considered up to now, the possibility that the real world is described by one of these states appears somewhat dim.

\footnotetext{
${ }^{1}$ We are assuming, here, that there is not some sort of (possibly cosmological) selection principle which picks out one or a few states; in this case, there is not really a discretuum of states. An example of such a principle is the hypothesis of cosmological SUSY breaking[11].
} 
One possible mode of explanation is to simply take a set of observed experimental facts, the gauge group, particle content and couplings of the Standard Model, the spectrum of fluctuations of the CMBR and the like, as given, and ask whether further predictions are possible. The second is to adopt an anthropic explanation. In the first case, as we will explain, it is essentially impossible to determine which is the "true vacuum" within the discretuum. It is necessary to ask what (if any) subset of states is consistent with the observed experimental facts and whether the distribution of some as yet unmeasured quantities is highly peaked about some values (this viewpoint has been stated most forcefully by Douglas). The flux discretuum would appear to be a natural framework in which to implement the anthropic principle[12]. Indeed, it is clear that in the context of the landscape, this is the only way in which to explain the value of the cosmological constant, and probably several other quantities of low energy physics. But we will see that in the flux discretuum, such a viewpoint will almost inevitably lead to predictions which are false.

We have previously presented [26] a variety of arguments against the utility of the anthropic principle in models of this type. In asking about anthropic vacuum selection, we distinguished two possibilities (what Dimopoulos has dubbed "habitability criteria"), which we will refer to as "Selection Principle A" and "Selection Principle B". Principle B selection principle asserts that any states which meet some very minimal criteria for the existence of life - the formation of structure in the universe, perhaps of stars, and the like, are acceptable. Selection principle A postulates the necessity for our own type of carbon based life. Principle B is a very reasonable constraint on mathematical theories of the universe, but is not very powerful. Principle A is potentially much more powerful, but requires one make assumptions which are very difficult to justify. Given a collection of low energy gauge groups and representations, we simply do not understand enough about complex phenomena in (the various possible analogs of )low energy nuclear physics, much less (exo)biology, to assert that life is or is not possible. One of our points will be that even if we implement some form of principle A, the landscape is likely to make many incorrect predictions.

In [26], we presented a variety of arguments against the utility of even principle $\mathrm{A}$ in models of the class exemplified by the flux discretuum. In this paper we modify and extend those arguments, explaining why the predictions of principle A within the framework of these models, are likely to differ widely from experimental values of many parameters.

Apart from these negative comments, we note that there is one interesting prediction that the flux vacuum might make: low energy supersymmetry. In particular, we explain why the 
vast majority of vacua with cosmological constant and gauge hierarchy required by principle A, are likely to exhibit some form of low energy supersymmetry. This is not necessarily a success; again, within the framework of principle A, our arguments suggest that the flux discretuum is not likely to agree with experiment. Some, though not all, of these observational problems are linked to the prediction of low energy SUSY.

We begin our discussion in the next section by critically examining the notion of the low energy effective Lagrangian, and the assumption that different solutions of the same long wavelength effective field equations are related to the same model of quantum gravity. Section 3 is devoted to a discussion of AdS and (primarily) meta-stable dS solutions of long distance effective SUGRA, presenting our detailed argument for the conclusions adumbrated above. In Section 4 we turn to phenomenological questions and the anthropic principle. Section 5 reiterates our conclusions. The system behind our exposition is to present a series of potential problems with the notion of a string theory landscape. These problems are ordered from fundamental to phenomenological. At each stage of the exposition, we ignore the objections of the previous stage, but the reader is certainly expected to keep them in mind.

\section{The proper use of effective lagrangians}

To explain our point of view about effective lagrangians in string theory, we will have to introduce some non-standard terminology. We will use the term long wavelength effective lagrangian to describe the object that is conventionally derived from quantum field theory or string Smatrices. In [15], one of the authors (TB) argued that, when supplemented with asymptotic boundary conditions in space-time, the long wavelength effective lagrangian in a model of quantum gravity also carries information about the high energy spectrum of the theory, via the properties of its black hole solutions. It was also argued that there were disjoint moduli spaces of models of quantum gravity, which could not be thought of as different states of the same theory. The latter notion (that of superselection sector in quantum field theory) depends on a separation between long wavelength and high energy physics, which is simply not valid in models of quantum gravity. We will use the term model of quantum gravity to refer to a single connected moduli space of S-matrices or boundary correlation functions ${ }^{2}$. The term theory of quantum gravity will be used to refer to an as yet only partially understood set of rules for constructing all sensible moduli spaces of models. The terms String Theory or M-theory should

\footnotetext{
${ }^{2}$ If there is a model describing stable asymptotically dS space, then it has a finite number of states and no precisely defined observables. Nonetheless, we would like to include this possibility in our list of possible models.
} 
be understood in this sense. We believe that there are many mathematically consistent models of quantum gravity, at most one of which describes the real world.

The main point of this section is that the same long wavelength lagrangian can describe the dynamics of different models of quantum gravity. This is best explained by examples. Consider the model called "M-theory Compactified on $T^{d}$ ", with $0 \leq d \leq 7$. It is defined by an S-matrix in $11-d$ asymptotically flat dimensions. The low energy dynamics of this model can be described by a long wavelength effective lagrangian. The lagrangian can be calculated by matching scattering amplitudes computed with it to amplitudes computed from the fundamental formulation of the theory. In terms of the lagrangian this corresponds to solving the classical equations of motion with scattering data of infinitesimal strength. The S-matrix is computed in terms of the asymptotic expansion of the solution in the strength of the scattering data. The effective action point of view is useful for computing the S-matrix as an asymptotic series in various ratios of length and energy scales.

The same lagrangian has classical solutions corresponding to 11 dimensional FRW cosmologies. In these solutions, the moduli of M-theory on a torus can vary with cosmological time. Spatial sections of the cosmology can have positive, negative or zero scalar curvature. It is clear that the observables (the S-matrix) of the well understood model do not generalize to these cosmological solutions. For any cosmological solution, the past or future asymptotic region ${ }^{3}$ is replaced by a singular space-time where all known approximate methods for solving M-theory break down. It may be that some of these cosmological solutions represent aspects of the physics of a well defined model of quantum gravity different from M-theory compactified on a torus. At present we do not have the tools to study this question, or to decide whether various cosmological solutions are all related to the same model. It seems likely that at least some of these solutions do not correspond to any sensible model. Thus, the existence of M-theory compactified on a torus as an S-matrix theory in asymptotically flat space, does not guarantee the existence of these cosmological models.

A similar example can be generated by studying M2-branes near conical singularities, whose near horizon geometry has the form $A d S_{4} \times K$, with $K$ a compact Einstein manifold. The geometrical moduli of $K$, become coupling constants in the family of CFT's which can be obtained in these limits. Each is a different model of quantum gravity. By studying nearly singular limits of $K$ we can generate examples of this type with a single light supermultiplet, much lighter than any of the other massive states of the theory. It is believed that the exact

\footnotetext{
${ }^{3}$ or both
} 
quantum dynamics of this system is described by a conformal field theory in $2+1$ dimensions. The long wavelength effective lagrangian is SUGRA coupled to the light scalar multiplet, with a negative cosmological constant. We can find other solutions of the same lagrangian, among them matter dominated FRW universes with Big Bang and/or Big Crunch singularities, as well as the Godel universe. Again it is clear that these are not all part of the same model of quantum gravity. The existence of the exact CFT description of the quantum dynamics of the AdS solution says nothing about the existence of quantum models which might be approximated by these other solutions.

The lesson of these examples is plain. Quantum gravity is not like quantum field theory, where a change in boundary conditions changes only a limited set of degrees of freedom of the system. In gravitational physics the asymptotic boundary conditions define the whole system. Our idea that different solutions of a long wavelength effective lagrangian are really part of the same theory is a relic of QFT intuition, and must be discarded. It follows that demonstrating the existence of an extra solution to a lagrangian derived from one well defined model of quantum gravity does not by itself imply that the solution corresponds to another well defined model.

These examples are consistent with the message of $[15,16]$, which is that the off shell effective potential is not a meaningful quantity in models of quantum gravity. It can at best be viewed as a heuristic tool, valid in some extreme regions of moduli space.

\subsection{The Effective Potential in String Perturbation Theory}

The arguments of $[15,16]$ and those based on AdS/CFT, depend on properties of black holes, which are non-perturbative in the string coupling. This might lead one to suppose that considerations based on the effective potential might be well defined to all orders in string perturbation theory when vacuum energies are of order the string scale or less. There is no evidence that this is the case. The effective potential, when it is non-zero, is an off shell quantity and we generally have no way to compute it beyond the first order in which it appears.

For many years, it was believed that the work of Fischler and Susskind[14] provided a

method for computing systematic corrections to the effective equations of motion in string perturbation theory. The idea was that divergences in integrals over the moduli space of Riemann surfaces would, for a general tree level string background, contribute anomalous terms 
to the BRST Ward identities for higher genus string computations:

$$
\sum_{i}<V_{1} \ldots\left[Q, V_{i}\right] \ldots V_{n}>_{g} \neq 0
$$

A non BRST invariant perturbation of the world sheet lagrangian, of order $g_{S}^{2 g-2}$ would be inserted to restore BRST invariance of the full sum of amplitudes, up to that genus.

This prescription appears to provide a way of correcting the equations of motion of fields which are massless at tree level, to all orders in string perturbation theory. Of course, these manipulations do not define an off-shell potential. They merely enable one to obtain quantum corrections to the on-shell condition. For massive fields, there is no analogous prescription, and we might not expect one. Most of these fields correspond to unstable excitations, which do not appear in the S-matrix. Even for stable massive modes, the possibility of doing nonlocal field redefinitions, with non-locality of scale $\sqrt{\alpha^{\prime}}$, makes the notion of effective potential ambiguous. As a consequence it is not at all clear how to use these perturbative methods to compute the properties of minima at finite values of the string coupling, as would be needed to discuss flux compactifications.

Even for massless fields, there is a technical problem ${ }^{4}$ with the Fischler-Susskind program. The one loop BRST anomaly produces a tadpole for the dilaton field. The linearized solution of the corrected dilaton equation of motion grows like two powers of the time. However, verification of the BRST Ward identity involves a partial integration in space-time. Thus, there is a space-time surface term in the BRST anomaly, which does not vanish even when we solve the Fischler-Susskind equations.

This might be attributed to a failure to solve the full nonlinear equations of motion. However in [17] two of the present authors argued that nonlinear solutions to the FS equations always replaced at least one of the two asymptotic boundary conditions with a finite time space-like singularity. Thus, the FS program does not give a controlled expansion of the string equations of motion. The small perturbative corrections become arbitrarily large at late or early times. We emphasize that this all occurs in a region where the string coupling is weak.

To summarize, even in perturbative string theory, we have no systematic definition of an effective potential. At this stage an analogy might be useful. When computing the amplitude for a quantum system to move between two points in configuration space over some time interval, one can often apply the WKB approximation, and do the computation in terms of a classical

\footnotetext{
${ }^{4}$ Pointed out to us by Eva Silverstein.
} 
solution of the equations of motion with boundary conditions that it hits the two points at the ends of the given time interval. It is certainly possible to calculate quantum corrections to the amplitude, but there is no known meaning to the quantum correction to this classical path ${ }^{5}$. The next correction to the amplitude sums over all deviations from the classical path.

The point of this analogy is that the effective potential in theories of quantum gravity may play a role analogous to the classical path between two points in ordinary quantum mechanics. In leading approximation it may be a device for determining the stability of a hypothetical model of gravity, but there may be no exact object in the theory to which it is an approximation. Much of the thinking implicit in discussions of flux compactifications depends on the notion that the effective potential is an exact object, for which we are presently able to find only approximate expressions. This line of thought might be completely wrong. We have no evidence from string theory or gravity that such an object exists.

In the remaining sections of this paper, we will ignore the caveats we have just enunciated. That is, we will explore hypothetical solutions to string theory which are motivated by finding minima of a low energy effective potential. The thoughtful reader will however, keep these remarks in mind as he follows our path through the hypothetical landscape of string theory.

\section{Exploring the Landscape of Flux Compactifications}

For definiteness, we will restrict our attention to compactifications of six dimensions in Type IIB string theory, which have been explored in great detail. One begins by searching for SUSic flux compactifications, using the GVW[18] superpotential in the presence of fluxes, branes and orientifolds $^{6}$. The fluxes are quantized, and the stationarity condition for the superpotential fixes the six manifold to be conformally Calabi-Yau, as well as fixing the dilaton and the complex structure moduli. In the classical approximation the Kahler moduli are left free because the superpotential does not depend on them. We obtain a no-scale SUSY breaking solution with vanishing cosmological constant. It is clear that corrections to the Kahler and super potentials will drastically change this scenario. The authors of [7] study a modification of the superpo-

\footnotetext{
${ }^{5}$ There is a quantum effective action, which is a generating functional for Green's functions. One can compute quantum corrections to the action and find modified "classical" solutions of it. But their purpose is to be expanded in powers of a source and generate Green's functions. They do not modify the path between a pair of points.

${ }^{6} \mathrm{~A}$ naive person might have imagined that it was inconsistent to include D-branes, which are singular sources with energy of order $\frac{1}{g_{S}}$ in string units. However, for SUSY preserving solutions, all terms of this order cancel exactly. For SUSY violating compactifications this issue must be revisited. We are not sure of the status of this issue at present.
} 
tential due to gaugino condensation, in models with only one Kahler modulus. This induces dependence of the superpotential on the Kahler modulus, and allows for a SUSY preserving minimum where the Kahler modulus is fixed and the cosmological constant is negative. This is the first indication of a landscape. By using the wide variety of fluxes available on a $C Y_{3}$ with large $b_{3}$ we can construct a plethora of SUSic AdS solutions of the effective field equations.

We consider this construction to be highly plausible, although there is no sense in which these solutions are part of the same model of quantum gravity as the solutions with vanishing flux and vanishing cosmological constant. There is nothing in the physics of these solutions which indicates a breakdown of long wavelength effective field theory (as long as the fluxes are chosen to be sufficiently large that all dimensions of the manifold are large compared to the string scale). Even effective field theory corrections to the cosmological constant are under control because of exact SUSY. There are only two disturbing aspects of this construction. Although much effort goes into guaranteeing that the string coupling is small, there is no systematic string perturbation theory around these solutions. Indeed, although we have invoked non-perturbative low energy physics to stabilize the Kahler modulus, there is no rigorous way of estimating the corrections, let alone a systematic machinery for calculating them. More generally, since for fixed topological numbers, the number and magnitude of fluxes that are allowed for compactifications to 4 dimensions are bounded from above, there is actually no expansion parameter which could be made arbitrarily small ${ }^{7}$

For the case of solutions with negative cosmological constant, a truly rigorous test of these proposals would be to discover the three dimensional CFT's which are dual to them. Progress along these lines was reported by Silverstein[10] at the Strings 2003 Conference in Kyoto. The basic idea is to use "flux-brane" transitions[13] to convert a flux compactification into a configuration of D-branes, and to study the world volume field theory of the resulting branes. It will be a $2+1$ dimensional quiver gauge theory. If the corresponding $3+1$ dimensional theory is not asymptotically free, one can imagine obtaining a perturbative zero of the $\beta$ function by cancelling the running of the coupling from its engineering dimension, with a large one loop quantum correction. Such perturbative fixed points cannot represent large radius AdS compactifications. However, in a similar situation in $3+1$ dimensions, the perturbative fixed points actually persist (at least for SUSic theories) into the strong coupling regime. One might hope that by varying the discrete parameters specifying the putative quiver duals to large radius

\footnotetext{
${ }^{7}$ The question of bounds on the flux number is somewhat subtle. The usual constraint arises from tadpole cancellations. In this relation, it would appear that by suitable choices of signs, some fluxes could be made arbitrarily large. As we will see, however, in these limits, the approxmimations of [7] break down.
} 
flux compactifications, one could find perturbatively accessible fixed points whose existence would lend support to the claim that the quivers indeed flow to fixed point theories.

Thus, although the notion of off shell effective potential does not have a meaning in models of quantum gravity, there are indications that the prediction of a discretuum of AdS vacua, which follows from this formalism, is plausible. Before discussing dS vacua, we revisit the analysis of the effective lagrangians which yield the supersymmetric AdS flux vacua.

\subsection{Detailed Analysis of the Discretuum}

In this section, we will review the analysis which suggests the existence of the flux discretuum. We want to focus on the question of the degree to which the existence of the flux discretuum, even within the framework of conventional effective field theory analysis, has been established. The points we will make here have all been made in [7], but they are worth emphasizing. In particular, we want to address the question of whether there is a small parameter which could justify the analysis.

The authors of [5] were interested in exhibiting warped vacua of string theory in a more or less controlled approximation. They found that, for fluxes located on a collapsing three cycle near the conifold point, one does obtain such warping. They also found that some of the moduli (the modulus associated with the deformation of the conifold, in particular) were fixed. In the approximation in which they worked, the superpotential for this modulus, $z$, along with the dilaton multiplet, $\tau$, was given by:

$$
W=(2 \pi)^{3} \alpha^{\prime}(M \mathcal{G}(z)-N \tau z)
$$

where

$$
\mathcal{G}(z)=\frac{z}{2 \pi i} \ln (z)+\text { holomorphic }
$$

This has a supersymmetric minimum where

$$
D_{z} W=\frac{\partial W}{\partial z}+\frac{\partial K}{\partial z} W=0
$$

which is solved by

$$
z \sim \exp \left(-\frac{2 \pi N}{M g_{s}}\right)
$$

If the ratio $N / M$ is large, then $z$ is very small. The corresponding space can be shown to be highly warped. The superpotential is exponentially small at this stage. 
They then noted that by including additional fluxes, it is possible to fix other complex structure moduli, including $\tau$. The resulting superpotential has the structure:

$$
W=(2 \pi)^{3} \alpha^{\prime}\left[M \mathcal{G}(z)-\tau\left(K z+K^{\prime} f(z)\right)\right]
$$

This has a supersymmetric stationary point,

$$
D_{\tau} W=\frac{\partial W}{\partial \tau}+\frac{\partial K}{\partial \tau} W=0
$$

when

$$
\bar{\tau}=\frac{M \mathcal{G}(0)}{K^{\prime} f(0)} \quad W=2(2 \pi)^{3} \alpha^{\prime} M \mathcal{G}(0)
$$

The resulting stationary point still yields a warped geometry, but now at the stationary point, $\langle W\rangle=W_{0}$ is no longer exponentially small; it is large in Planck units; in fact, including fluxes to fix the other complex structure moduli, its value in Planck units is typically of order some large flux number. We will see however that special configurations can have relatively small $W_{0}$. We believe that at best, for reasons which we will now explain, the analysis can be justified only for these special states.

It should be noted here that in the best of cases there is no systematic computation which justifies this calculation. The flux numbers cannot be taken arbitrarily large, for fixed $C Y_{3}$. Still, the fact that these numbers can be quite large in particular examples creates the hope that the results are meaningful.

In this approximation, the radial modulus is not fixed. The authors of [7] argued that various processes, such as gaugino condensation in the low energy theory, would modify the superpotential, adding terms of the form

$$
W=W_{0}+e^{i \rho / b}
$$

where $\rho$ is the radial multiplet, and $b$ is a constant. At the stationary point, $e^{i \rho / b} \sim W_{0}$. But we have noted that $W_{0}$ is typically large, so $\rho$ is small, and the analysis is not self consistent. The spirit of [7] is that, given the huge number of possible choices of flux, $W_{0}$ will be small for many choices. For these, $\rho$ will be large. In addition, while the masses of the Kaluza-Klein modes are of order $1 / R^{2}$, the masses of the dilaton and the complex structure moduli are of order $1 / R^{3}$ (though possibly times large flux factors), and the mass of $\rho$ will be of order $\frac{\left|W_{0}\right|^{2}}{R^{2}}$. So the picture of first integrating out higher dimensional physics, followed by the complex structure moduli, followed by $\rho$, is consistent. 
We should note, however, a potential difficulty. The masses of the complex structure moduli are typically enhanced by a factor of flux-squared, i.e. they are of order $\frac{N^{2}}{R}$ relative to the Kaluza-Klein masses. So one requires very large $R$ if these are to be small relative to the Kaluza-Klein scale. More precisely, this ratio is of order

$$
\frac{m_{c s}^{2}}{m_{k k}^{2}} \sim \frac{K^{2}}{\rho \tau}
$$

where $K$ is a typical flux number. So if $\tau \sim K$, then we require $\rho \gg K$. Recall that $e^{i b \rho} \sim W_{0}$. So $W_{0}$ must be extremely small. For small $W_{0}$, the distribution of $W_{0}$ 's might be expected to behave as 8

$$
P\left(W_{0}\right) \propto \frac{W_{0}}{M_{p}^{3}}
$$

So only in an exponentially small fraction of states is the analysis which leads to the flux vacua completely under control.

It should be noted at this point that if one tries to make some fluxes extremely large, consistent with the tadpole cancellation condition, the low energy field theory approximation certainly breaks down.

The authors of [7] went on to suggest one possible mechanism for supersymmetry breaking. They argued that if one includes $\overline{D 3}$ branes in these compactifications, they would naturally sit near the (resolved) singularity, and would have exponentially small tension due to warping. Their presence breaks the supersymmetry, so one would have a small breaking of supersymmetry. The actual cosmological constant depends on an interplay between $W_{0}$ (determined randomly) and the exponential warp factor. These are not correlated in any particular way, but in some subset of states, one might expect (at least at the level of the effective action) to find supersymmetry breaking with a small, positive cosmological constant, as a result of cancellations.

There are other ways in which one might imagine supersymmetry breaking to arise. For example, the low energy theory might be a theory in which supersymmetry breaks dynamically ${ }^{9}$. Again, using conventional effective field theory reasoning, one might expect to find,in some

\footnotetext{
${ }^{8}$ Kachru (private communication) has explained this heuristically by noting that, if one thinks of the fluxes as forming a vector, $W_{0}=\vec{N} \cdot \vec{a}$, where $\vec{a}$ is a set of constants, which for large $\rho$ are roughly independent of $\rho$. So $W_{0}$ can be thought of as the dot product of two vectors in a high dimensional space; calling the azimuthal angle $\theta$, one is interested in the distribution of $\cos (\theta)$ around $\theta=\frac{\pi}{2}$. This picture cannot be taken too literally, since the the $a$ 's, for example, depend on the complex structure.

${ }^{9}$ This raises puzzling issues, which will be dealt with elsewhere[19]
} 
fraction of vacua, a small, positive cosmological constant, as a result of cancellation between physics at two, a priori unrelated, scales.

One question here is what the role of supersymmetry is in this analysis. After all, in some fraction of states, the radii might be expected to be large and the cosmological constant small, simply by accident. We will return to this question shortly and discuss whether the flux discretuum might lead to a prediction of low energy supersymmetry.

\subsection{Vacuum Tunneling and the Existence of Meta-stable dS States}

Conventional discussions [1, 2] of flux compactifications envisage vacuum tunnelling between solutions with different values of the cosmological constant. This is often assumed to be the mechanism which dynamically implements the anthropic principle. The universe jumps around between vacua until it finds itself in an anthropically allowed one, at which time we observe it.

In fact, as was recently emphasized in [20], the original Coleman-De Luccia paper on vacuum tunnelling in the presence of gravity implies important modifications to this idea. These modifications are amply confirmed[20][15] by the modern interpretation of the quantum mechanics of AdS universes in terms of conformal field theory. We will first list them and then explore their implications for flux compactification models.

- There are no semiclassical tunnelling amplitudes into AdS space times. When there is a non-vanishing amplitude to tunnel into the basin of attraction of a negative cosmological constant minimum, the post-tunnelling classical evolution is a negatively curved FRW cosmology, which undergoes a Big Crunch.

- Amplitudes for tunnelling between any pair of asymptotically dS universes, are finite. This is true in the sense that the post-tunnelling solution inside a cosmological horizon volume, asymptotes to the dS space with appropriate cosmological constant. The global structure of the post-tunnelling solution is not that of $\mathrm{dS}$, but this may be unimportant, since no observer can see more than a horizon volume. The ratio of probabilities for jumping in the two directions is governed by a law of detailed balance with the entropy rather than the free energy in the exponent. This is consistent with thermodynamics if most of the states of dS space have energies below the dS temperature.

- There are no semiclassical tunnelling amplitudes between dS space and asymptotically flat space. When there is a non-vanishing amplitude to tunnel into the basin of attraction of a 
region of field space with vanishing cosmological constant, the post tunnelling evolution is a negatively curved FRW solution, which is not asymptotically flat. It is often nonsingular in the future, but has a Big Bang singularity in its past.

Thus, while the idea of jumping between dS states might make sense in the semiclassical approximation, jumping to regions with non-positive cosmological constant always involves a breakdown of effective field theory.

We suspect that the Big Crunch singularities are a more serious problem, and do not occur in well-defined models of quantum gravity. This suspicion is based on the AdS/CFT correspondence. An asymptotically AdS universe is described quantum mechanically by a conformal field theory, and the cosmological constant controls the asymptotic density of states in the CFT. Such a theory is by definition stable, and has a conformally invariant vacuum state. Thus, although long distance effective Lagrangians can exhibit tunnelling between AdS vacua and Big Crunch FRW universes, this does not appear to be a feature of consistent theories of quantum gravity. Of course, this does not imply that the analogous tunnelling solutions for non-negative cosmological constant cannot occur, but it is suggestive.

If we suppose that tunnelling between $\mathrm{dS}$ minima can occur as suggested by the semiclassical approximation, the above discussion suggests a potential instability for the whole complex of metas-stable dS flux vacua. Suppose that in a consistent model, one cannot tunnel into a Big Crunch. If any meta-stable dS state can tunnel to any other, and if one of them can tunnel to a Crunch, then the whole system might be inconsistent.

The reader may object that if the amplitudes to tunnel to disaster are much smaller than those to tunnel to a zero cosmological constant region (which always exists in flux compactifications) then they should be ignored in the semi-classical approximation. We present the following quantum mechanical model as a counter-example: Consider the motion of a particle in a plane under the influence of a potential

$$
V(r, \theta)=\frac{\left(r^{2}+a^{2}\right)}{\left(b^{2}+r^{4}\right)} \cos ^{2} \theta+\epsilon r^{4} \cos ^{5} \theta
$$

There is a meta-stable minimum at the origin, with two semiclassical modes of decay. Let us take $\epsilon \ll 1$. Then there is a stable minimum at $\theta=0$ and $r \sim \epsilon^{-1 / 6}$. The potential is unbounded from below when $\pi \leq \theta<2 \pi$ and the Hamiltonian does not exist as a Hermitian operator. However, for small $\epsilon$ the tunnelling amplitude to the unbounded region goes to zero exponentially, while 
that to the stable minimum in the forward direction goes to a constant. If all we knew how to do was the semi-classical physics of this model, we might have mistakenly ignored this sign of catastrophic instability. In ordinary quantum mechanics, semiclassical tunnelling calculations explore the potential surface. Thus, even when a given tunnelling amplitude is too small to be included in a systematic expansion scheme, it may reveal true instabilities of the system. ${ }^{10}$.

Even if this potential instability turns out to be a chimera, the semiclassical discussion of meta-stable dS states still contains a potential problem. One cannot understand a meta-stable state without understanding the stable system of which it is a temporary excitation. The only plausible candidate for a semiclassical description of that stable state is, in the context of flux compactifications, a negatively curved FRW solution with moduli moving on the Dine-Seiberg region of the potential. This is the space-time that appears in the analytic continuation of the instanton for meta-stable dS decay. There are good and bad aspects of this suggestion.

It is often assumed, by analogy with quantum field theory, that a meta-stable dS state could be an excitation of an asymptotically flat space-time. In field theory this is consistent. The post-tunnelling solution indeed asymptotes to flat space with constant field at the stable minimum. Furthermore we can excite arbitrarily large regions of the stable flat space into the positive energy density minimum. Neither of these statements is true when gravity is dynamical. Guth and Farhi [21] demonstrated that if one tries to excite a field coupled to gravity into a meta-stable minimum of its effective potential, one instead creates a black hole. Consistent with this observation, the post-tunnelling solution does asymptote to an FRW cosmology ${ }^{11}$. Furthermore, it is at least plausible (given the quantity of literature on old inflation) that a negatively curved FRW space-time can, at sufficiently early times, be excited into a meta-stable dS state. This question deserves much more careful study, and is the heart of the issue. To study it, one would have to develop a quantum theory of negatively curved Big Bang FRW universes and determine whether it really exhibited meta-stable dS states.

The real fly in the ointment is that the possibility of exciting the meta-stable dS state depends precisely on the fact that the FRW solution evolves from a Big Bang singularity. That is, it is because of the increase of energy density at early times that we believe that we can easily go over the barrier to the meta-stable state. It would be disingenuous to ignore the fact that

\footnotetext{
${ }^{10}$ Susskind argues that the Big Crunch singularities are innocuous because there are causal patches which are causally disconnected from them. Thus the singularities should be viewed as black holes from the point of view of observers in these patches[24]

${ }^{11}$ If the zero c.c. minimum is a stable minimum at a finite point, this will be a matter dominated FRW model. If, as is the case in string theory, the minimum is at infinity, then we get a kinetic energy dominated FRW with fields in the Dine-Seiberg region of moduli space.
} 
this is happening because we are approaching a region where effective field theory is breaking down.

We are quite sympathetic to the idea that Big Bang singularities (or their proper resolution) may be part of some consistent models of quantum gravity. After all, our own universe appears to have such a singularity in its past. However, without a thorough development of the full quantum theory of such systems, it seems premature to conclude that the landscape of metastable dS minima is really a feature of string theory. It is only by finding a full quantum description of the FRW solution that we can hope to calculate corrections to the effective field theory description of the meta-stable minima.

This brings up the question of how the meta-stable dS minima are related to mathematically well defined observables of the putative stable FRW solution. The asymptotic future of the latter is a smooth space-time where asymptotic data can be formulated. Thus we can imagine that the quantum theory of such a space-time is defined by an S-matrix or S-vector[22] relating such future asymptotic states to a basis of states defined at the Big Bang. The term S-vector refers to the possibility that only one state is allowed at the Big Bang (or in [23] that all states are gauge equivalent there). If there is indeed an S-matrix, one can imagine picking out a particular meta-stable dS state and tuning the scattering data to find an S-matrix element or elements in which it appears as the dominant resonance. The relation of these S-matrix elements to local measurements performed by meta-stable observers in the meta-stable dS state is obscure, but presumably if we discover the appropriate quantum theory we will be able to work it out. 12

It is much less clear what to do if the system only has an S-vector. Then one cannot tune initial conditions and any amplitude would be some complicated superposition of contributions from the different dS states.

In summary, the landscape of meta-stable dS minima suffers from a potentially catastrophic instability. In addition, the candidate for the stable state into which it decays suffers from a Big Bang singularity. One cannot conclude that the landscape exists without understanding these singularities, not something we can do with the effective field theory techniques we currently have at our disposal. One will have to find a more fundamental definition of these states in order to calculate any of their properties beyond the effective field theory approximation.

\footnotetext{
${ }^{12}$ Susskind has suggested that we interpret the Big Bang of this solution as the expanding phase of a Big Crunch-Big Bang solution. The metastable dS vacua appear as resonances in an S-matrix for this time symmetric system. In our opinion, one must resolve the singularity to understand this system [24].
} 
We emphasize that this issue is connected with the question of parameters, which we asked above. In order to assess whether an expansion parameter is small enough for us to believe the predictions of a leading order calculation, we must have some understanding of the nature of the exact model to which the leading order is an approximation. For meta-stable dS minima, this exact model would be the theory of the S-matrix or S-vector in an FRW space-time with negative spatial curvature and a Big Bang singularity. It is only when we have some clue to the correct formulation of the quantum theory of such systems that we can really begin to assess whether meta-stable dS minima exist. Without such an exact definition, we cannot compute even one step beyond the leading order in the putative expansion.

In the remainder of this paper, we will assume that the landscape exists and examine the question of whether it is likely to lead to a description of the real world. An immediate question to ask is how the cosmological constant would be explained in such a scenario. Controllable examples are expected to have a cosmological constant which is smaller than string scale, but not much smaller than the scale of SUSY breaking. However, the huge size of the discretuum suggests the possibility that the mechanism of $[1]^{13}$ for obtaining a tiny cosmological constant and a large SUSY breaking scale might be operative. This explanation relies on the anthropic principle[12]. In a huge collection of meta-stable dS vacua, a minimum with a small value of the cosmological constant might be picked out by the anthropic principle. In the context of flux compactifications, it is implausible that there will be only a few minima satisfying the anthropic constraints on the cosmological constant - many other parameters of the long wavelength effective field theory are likely to vary discontinuously as one jumps from one minimum with an anthropically allowed c.c., to another. Much of the remainder of this paper will be concerned with the likelihood that anthropic predictions for low energy physics actually fit the data.

\section{Scientific Explanation in the Discretuum}

There are two possible modes of scientific explanation in the discretuum. The first is to simply to ask whether there are states in the discretuum with properties identical to those of the Standard Model and observational cosmology, and to ask whether the physics of the ensemble of such states is sufficiently similar that one can make further predictions[9], for as yet unmeasured quantities ${ }^{14}$. At the crudest level, the problem is one of counting. In order to explain

\footnotetext{
${ }^{13}$ An older variant of this idea appeared in [25] .

${ }^{14}$ Another problem here is the inevitable imprecision of quantities associated with a particular state in the discretuum, due to its finite width. For example, all energy levels should be broadened. Is this consistent with an enormously precise tuning of the value of the c.c. associated with this state?
} 
the cosmological constant alone, one needs of order $10^{63}-10^{120}$ states (depending on one's assumptions about supersymmetry and supersymmetry breaking). Additional requirements, such as a suitable hierarchy, the correct values of the quark and lepton masses and mixings, the correct amount of inflation, and so on, are likely to increase this by an enormous factor, quite possibly just as large or larger. Refererence [7] provided some estimates of the number of flux vacua, and [9] examined some prototype counting problems, which suggest that there could conceivably be enough flux vacua.

The problem of actually determining the correct ground state is hopeless, from this viewpoint. It is not clear that there is any sort of small expansion parameter in the flux vacua. If there is, it is unlikely to be smaller than a part in 100. This means that, if one works say to tenth order in this parameter (far more precise than any precision QED calculation) one would calculate the cosmological constant, at best, to an accuracy more than 40 orders of magnitude less fine than the observed value of the dark energy. In other words, at this stage of the computation, there must be at least of order $10^{40}$ states with properties otherwise identical to those of the Standard Model; determining which of these described nature would require an impossibly difficult calculation.

Instead one would want to ask whether the bulk of these $10^{40}$ states had some other characteristic properties, which could lead to predictions for experiments that were not used as input for determining the allowed ensemble of states. We will speculate below that low energy supersymmetry might be such a prediction.

The alternative mode of explanation is anthropic. This clearly raises many of the same issues that we have just discussed. The hope, however, is that our presence as observers provides an explanation of some of the experimental facts which we previously took as input. The possibility that such considerations might explain the value of the cosmological constant has perhaps received the most attention through the years, but one might hope to understand many other issues: the gauge groups of the Standard Model, the values of some of the quark and lepton masses and the gauge couplings, and so on. Within the flux discretuum, as we will explain in more detail below, all of the parameters of the Standard Model vary among the different states. As a result, in this viewpoint, the parameters of the Standard Model are either anthropic or random. As we will discuss, it is unlikely that even principle A can explain the values of the Standard Model parameters, or all of the observed cosmological parameters. On the other hand the anthropically unexplained parameters do not appear random, i.e. typical of the distributions which seem likely to emerge from the flux discretuum. This leaves the 
possibilities that the discretuum fails to reproduce the world we see, or that some additional, rational explanation is required. As we will discuss, one possibility is that there is a symmetry explanation. For example, we could imagine that a light $u$ and $d$ quark are required by anthropic considerations, but that many more states have light quarks as a result of symmetries than simply by accident (though crude estimates of the fraction of states in the discretuum which possess discrete symmetries suggest that this is not likely to be the case).

\subsection{An Aside on Continuous Moduli Spaces}

As an illustration of the issues raised in the previous section, let us first consider a set of states which we have every reason to believe make sense in quantum gravity. These are moduli spaces with more than four supersymmetries, in various numbers of dimensions greater than or equal to four. In these cases, the supersymmetry forbids any potential on the moduli space. As we mentioned earlier, string duality has shown convincingly that there are no non-perturbative difficulties with the construction of these models.

So what are we to make of these? In our first paradigm, we would simply assert that nature is four dimensional, with less than four supersymmetries. This is a disappointing retreat, and it is in this context that the anthropic principle may be necessary and even appealing. Certainly carbon-based life will not arise in these states, so principle A adequate to rule out these states. It seems quite likely that even principle B might also successfully explain why life can only exist in a state with no supersymmetries (possibly four approximate supersymmetries). Truly establishing this requires careful thought, but there are likely to be a number of difficulties with creating any complex structures in such states.

1. Conventional stars will not exist, even assuming an unbroken $U(1)$.

2. Planets, if they exist, will be very unlike those we see in the universe.

3. It may be difficult, given the lack of moduli potentials, to understand inflation in these states.

One can, of course, put forward possible scenarios which would evade each of these problems. But it seems to us likely that an application of principle B might be adequate to understand this most basic fact. 


\subsection{Extremely Light Scalars?}

While we are on the subject of the anthropic principle and light or massless scalars, let us recall a proposal for implementing the anthropic principle, which involves an extremely light scalar field $[28,29,30,31]$. The basic idea is very simple. Suppose, for definiteness, that at very low energies, the effective lagrangian for this particle is given by:

$$
\mathcal{L}=\frac{1}{2}\left((\partial \phi)^{2}-m^{2} \phi^{2}\right)+\Lambda .
$$

The time evolution of this field, in an FRW universe, is given by:

$$
\ddot{\phi}+3 H \dot{\phi}+m^{2} \phi=0
$$

If $m \ll H$, then the field $\phi$ is essentially frozen.

Now one imagines that during an extended inflationary period, there are many regions of the universe with differing values of $\phi$. If $\phi$ can vary over a huge region of field space, there will be some regions where the effective cosmological constant is small enough to satisfy anthropic bounds, and comparable to the dark energy today. Note that huge here means that $\phi$ is at least as large as (assuming that $\Lambda$ in eqn. 11 is of order $\mathrm{TeV}^{4}$ )

$$
\phi=M_{p}\left(\frac{M_{p}^{2}}{\mathrm{TeV}^{2}}\right)
$$

It is interesting to ask whether fields with such properties exist in string theory. Experience with string loop calculations indicates that conventional ideas about naturalness of scalar masses are always applicable. In other words, it is highly unlikely that one has massless scalars with masses much less than the scale of supersymmetry breaking (apart from a possible loop factor or two), except for axion-like objects.

So $\phi$ is most likely an "axion", i.e. a field with some periodicity. This axion must have a decay constant enormous compared to the Planck scale. While no one has proven a theorem that the Planck scale is an upper bound on axion decay constants, extensive searches[32] have yielded no examples of parameterically large decay constants. So it seems unlikely that this solution of the cosmological constant problem is implemented in string theory (the only theory where this question can be sensibly addressed). 


\section{The Anthropic Principle in Flux Compactifications}

In this section, we discuss the anthropic principle as possibly realized in flux vacua, and its limitations, in greater detail.

\subsection{The Anthropic Principle as a Datum}

All currently accepted physical theories require some phenomenological input. Our recent enthusiasm for string theory as the theory of everything has given rise to the hope that the only necessary inputs are the basic dimensionful parameters which define the conversion between socially defined scales of measurement and the fundamental units of mass, length, time and action. This is not necessarily the case, and the existence of mathematically consistent, disconnected, models of quantum gravity suggests that it is not the case. In this eventuality, we will have to supply other data as input, in order to decide on our theory of the world.

A charitable view of the anthropic principle is that it is simply a novel way of constraining theoretical speculations by fixing a single complicated piece of data about the world. The hope of the anthropically minded is that this single piece of complicated data will constrain many if not all of the simpler parameters which appear in the mathematical formulation the theory of the world.

In a previous paper, Motl and two of the authors[26] criticized the use of principle A (the insistence on carbon based life) as overly parochial. That criticism was based on a rather different view of the meaning of the anthropic principle. It seems tautological that physical theories which cannot give rise to complex life forms that could observe their consequences, will never be observed. This observation seems to make the anthropic principle an axiom, rather than a radical hypothesis. However, currently, we cannot derive the existence of life from fundamental physics. Furthermore, fundamental physics as we understand it today seems to allow for the possibility of many theories which resemble the standard model's gross features (abelian and non-abelian gauge groups, chiral fermions). It seems unlikely that we could ever discover (by pure thought! ) which of these potential low energy lagrangians could give rise to complex life forms. If one adopts only this tautological, but very weak, version of the anthropic principle (the biothropic principle), it is clear that current technology can only hope to determine a small number of parameters by anthropic arguments. Probably the only prediction of the biothropic principle is a relation between the cosmological constant, the amplitude of 
primordial density fluctuations, and the dark matter density at the beginning of the matter dominated era, which guarantees that galaxy formation is possible. Even "stellar astrophysics" and the details of "nuclear physics" are beyond our capabilities for general gauge groups and representations.

However, from the more modest point of view articulated above, principle A seems more reasonable. Indeed, the attraction of this principle for its advocates is that it appears to fix many physical parameters close to their observed values. It can also rule out many of the highly symmetric models of quantum gravity, with which string theory provides us. The existence of light photinos with relatively strong coupling, and degenerate bosonic partners for all fermions, suggests that in an exactly SUSic theory, the standard model would not give rise to conventional nuclei and atoms, but rather, Bose condensed clumps of the superpartners of electrons and nucleons ${ }^{15}$. In this more modest view, once we have accepted the existence of the meta-stable dS states implied by string theory flux compactifications, it would seem that the principle A might be an acceptable way to explain why we experience a particular one of these states.

The key question about principle A thus becomes whether it is a more economical way of constraining our models of the world than other pieces of data that one might use to the same end. In what follows, we will be examining this question in the context of flux compactifications of string theory. The parameter which cries out for an anthropic explanation in this context is the cosmological constant, since the meta-stable dS vacuum energies in these scenarios are not typically small enough to be compatible with the data. It was argued by Bousso and Polchinski $(\mathrm{BP})[1]$ that with a large enough range of values of a large enough collection of fluxes, it was natural to have some meta-stable minima with vacuum energy on the order of that which is indicated by cosmological observations. Subsequent work has tried to demonstrate, with some success, that the requisite number of vacua and fluxes exists in string compactifications. One might then argue that anthropic considerations will pick out a value of the cosmological constant consistent with observation.

We will discuss below the question of whether the anthropic prediction of the cosmological constant in these models is successful. Here we only want to note that if there are any metastable dS solutions consistent with the constraint, there are likely to be many. Within the BP ground rules, one would re-introduce fine tuning if one tried to insist that there were $\mathcal{O}(1)$ states

\footnotetext{
${ }^{15}$ Above, we have suggested that versions of this argument might be valid for a large class of models of low energy physics.
} 
which satisfied the anthropic constraint ${ }^{16}$. Thus, there will be many minima, with a variety of low energy effective theories, which satisfy the anthropic constraint on the cosmological constant.

One of the disappointing things about the principle $\mathrm{A}$ is that many aspects of the choice of low energy gauge group will be dictated by this principle, rather than determined dynamically. If we simply view the principle as a complicated datum this disappointment might turn into a virtue - our single piece of input might determine not only the cosmological constant, but also the structure of the standard model. However, as we will see in the next few sections, this is unlikely to be true. Principle A is not strong enough to determine the gauge group at the weak scale.

\subsection{The Renormalization Group and the Anthropic Principle}

To understand the utility of the anthropic principle as a piece of data, we must carefully separate it from other data that have been used to fix our current understanding of physics. Anthropic arguments involve physics at scales of a few hundred $\mathrm{MeV}$ or less. Apart from the parameters in the effective lagrangian at this scale, they depend on boundary conditions which are determined by high energy physics. These are the primordial density fluctuation spectrum, the baryon asymmetry, the density of dark matter at the beginning of the matter dominated era, and the fact of proton stability . Primordial element abundances are not terribly important, nor are any but the grossest properties of dark matter necessary to the formation of galaxies. The elements that concern us as humans were formed in stars. For anthropic purposes, star formation does not have to be such as to reproduce the observed stellar populations, but merely such as to produce a reasonable number of stars like our Sun. The question of what a reasonable number is constitutes a problem in exobiology which has not yet been solved.

For anthropic purposes we need only to have a nuclear physics reasonably like the existing one. Indeed, most detailed properties of elements heavier than carbon (or at most iron) are not important. They must only be such as to supply a level of radioactivity sufficient to fuel geological processes, and perhaps to catalyze an appropriate mutation rate for DNA.

Within the context of string theory, it is plausible to assume that the origin of nuclei comes from a strong gauge interaction, but there are lots of possibilities for obtaining particles

\footnotetext{
${ }^{16}$ This is true even if we consider the anthropic constraint as a constraint on the cosmological constant with all other parameters fixed at their observational values. In the true multiparameter fit, it would require even more tuning.
} 
like the proton and neutron. The simplest of these is the $S U(2 N+1)$ generalization of QCD with two light flavors. Note that the heavier families of quarks and leptons do not participate in anthropic considerations ${ }^{17}$. It is only the crucial dependence of stellar properties on the marginal stability of deuterium (and perhaps a few other poorly understood nuclear facts) that might lead one to believe that the value of $N$ was crucially important.

Nuclear physics at the level of anthropic precision, depends even less on the weak interactions. They enter only as four fermi operators and it is unlikely that even the $V-A$ structure is crucial. It is hard to decide this, because our ability to calculate nuclear properties is limited, but the fact that it took twenty years, and high precision experiments to establish the $V-A$ theory suggests strongly that the existence and gross properties of carbon, nitrogen, oxygen and iron nuclei would not be changed if the weak interactions were slightly different.

This is even more evident for the neutral current weak interactions. Their importance to anthropic arguments is limited to their role in supernova explosions. However, to the extent we understand this, a wide range of additional neutrino interactions would serve the same purpose.

More generally, there is a wide variety of modifications of the standard model of electroweak interactions, with new particles at the $50-100 \mathrm{GeV}$ scale, all of which are both ruled out by experiment, but compatible with the anthropic principle. These include models which can be derived from string theory e.g. intersecting $D 6$ brane "standard-like" models. Here we make the assumption that the exotic particles in these theories have masses which make them observable in current experiments but not in nuclear physics.

Other crucial anthropic features of the standard model are the proton neutron mass difference and the electron mass ${ }^{18}$. These involve the least understood part of the standard model, the light quark and lepton mass matrices. We will argue below that it is difficult to understand many features of these mass matrices in the context of flux compactifications. Here we only note that it is easy to satisfy the anthropic constraints on these masses in many extensions of the standard model which are ruled out by experiment. Thus anthropic arguments give us no way to distinguish between such models.

To sum up, the renormalization group implies that even principle $\mathrm{A}$ is not a powerful piece of data for discriminating between the behavior of different models at energy scales of order

\footnotetext{
${ }^{17} \mathrm{~S}$. Thomas has noted a possible exception. A muon with just the right mass gives us cosmic radiation which penetrates the atmosphere. One might speculate that this is important for evolution. On the other hand - given our lack of understanding of the value of the muon mass, one can easily imagine other models which give rise to a particle with the necessary properties.

${ }^{18}$ We take for granted the fact that we need a photon with the right value of the fine structure constant.
} 
$100 \mathrm{GeV}$. Anthropically interesting physics depends on the physics at these scales only through a few effective parameters. Flux compactifications appear able to generate a wide variety of gauge groups and matter representations, as well as a near continuum of values of couplings. It seems unlikely that even the requirement of carbon based life will be able to pick a unique solution from this large collection. For example, even if the gauge group and matter content is correctly selected, it is unlikely that anthropic considerations determine these parameters with sufficient accuracy that they are sensitive to the values of the $c, b$ and $t$ quark masses. If this is correct, and flux compactifications exist, then it appears that string theory, even when coupled with principle A, does not give definite predictions for physics at the electroweak scale. Indeed, it is likely to give rise to many anthropically allowed models which are already inconsistent with experiment. Of course, given our lack of knowledge of the landscape of flux compactifications, one can still argue that one might find only one minimum with the right value of the c.c. and approximately the right nuclear physics. Our observations should thus be viewed as a challenge to this set of ideas, rather than a no-go theorem.

There appears to be one possible loophole in this argument. Some parts of nuclear (and consequently stellar) physics appear very sensitive to small changes. We are thinking particularly of the margin stability of deuterium and the proton-neutron mass difference as low energy properties that dramatically affect nuclear and stellar physics. It is possible that a unique solution could be picked out by these sensitive properties. In order to verify this conjecture we would have to understand the whole collection of flux compactification models which give a more or less standard nuclear physics, and calculate the deuteron binding energy and proton neutron mass difference in each of them. The task of the anthropic flux compactifier is not an easy one.

One always emphasizes to one's students that the renormalization group is not a group but a semi-group. The burden of this subsection has been that this well known fact implies that principle $\mathrm{A}$ is not a terribly powerful piece of data for discriminating physics at the $100 \mathrm{GeV}$ scale. We will try to order our subsequent remarks about the anthropic principle in approximate "RG order" . That is, we will assume that some combination of anthropic arguments and knowledge of the flux landscape has successfully reproduced some aspects of physics at a given energy scale, and then explore the utility of these arguments for probing physics at higher energies. Our next step is to assume that the standard model gauge group has been fixed by these considerations and ask what further aspects of the standard model lagrangian will be simply explained by them. 


\subsection{Bottom Up Approach to the Phenomenology of the Discretuum}

The discussion of the renormalization group in the previous section suggests a "bottom up" approach to the phenomenology of the discretuum. Consider, first, the parameters of the effective lagrangian well below the QCD scale. At the smallest energy scales, we have the cosmological constant. At higher energies, we have the electron mass and the electromagnetic coupling (and the proton mass). We can well imagine that strong selection effects might choose among these. At still higher energies we encounter the parameters associated with nuclear and low energy QCD interactions. These include the number of colors, the $u$ and $d$ quark masses and the scale of QCD. It is easy to imagine that strong anthropic effects would account for the $u$ and $d$ quark masses. For example, if the $u$ quark were heavier than the $d$ quark, the mass of the neutron would be lower than that of the proton. Similarly, if the $u$ and/or $d$ quarks had their "natural" values, of the same order as the weak boson masses, then nuclear physics as we know it would not exist. This constitutes an anthropic argument for the small values of these masses, which are commonly believed to result from softly broken discrete symmetries. In the flux vacuum context, this is welcome, because, as we will see, discrete symmetries are broken at a high scale in most of these states. Similarly, the value of the QCD scale relative to the electron mass might be fixed by anthropic considerations in molecular physics.

Stellar processes are sensitive to the precise values of the above mentioned quantities. These are probably necessary to explain the gauge group of the strong interactions itself. As we have already remarked, if there are two light quarks, we might well imagine that nuclear physics is not qualitatively different for different values of $N$, and that for suitable quark masses it is even quantitatively almost the same. Only a very strong version of principle A, presumably related to the properties of deuterium, are likely to explain why the gauge group is $S U(3)$ and not something else.

However, there is one other parameter we encounter at this stage, which even principle A is not likely to explain: the QCD angle, $\theta$. It is hard to see an anthropic argument that $\theta$ is small, much less bounded by $10^{-9}$. Moreover, in the flux vacua, there is typically no light axion. Recall that the complex structure moduli were fixed, in a supersymmetry conserving fashion, at scales well above the supersymmetry breaking scale. The radius, $\rho$, was fixed by the superpotential:

$$
W=W_{0}+a e^{-b \rho} .
$$

So the real part of $\rho$, which we might hope to identify as an axion, in such models, gets a mass 
of order the scale of supersymmetry breaking, and is irrelevant to the strong CP problem. In general, obtaining light axions once supersymmetry is broken poses various difficulties[33]. It is clear that a "generic" flux vacuum does not readily yield an axion. Whether some special subset (e.g. with discrete symmetries) might yield one is an interesting question. Conceivably, the axion might be an accidental consequence of, say, discrete symmetries which are also responsible for the smallness of quark and lepton masses. However, we have already noted that there is a conflict between flux vacua and discrete symmetries.

Alternative solutions to the strong CP problem include a massless $u$ quark. Many theorists would argue that this possibility is now ruled out both by effective chiral lagrangian arguments and explicit lattice computations. But even if we are willing to contemplate it, it seems unlikely that this will arise in flux models, for the same reason that it is hard to understand light quarks and leptons: the absence of discrete symmetries in generic flux vacua. Alternatively, as in the models of Nelson and Barr[41], one could contemplate that the possibility of "spontaneous" CP violation, with $\theta$ small as a consequence of accidental features of the model. But given that typical fluxes violate $\mathrm{CP}$, it is hard to imagine that this could arise, again, in any but a modest subset of these states.

There is only one way that we can imagine resolving this conundrum. We have already argued that anthropic reasoning forces the two lightest quarks to have masses close to their observed values. Suppose we were to find that in the vast discretuum, this was achieved most often in states with appropriate discrete symmetries (rather than simply randomly). Note that this is very different from anthropic arguments for the cosmological constant, or the weak scale. In those cases we abandon the traditional search for symmetry arguments to explain small numbers, and simply throw away all states which do not satisfy the anthropic bound. Now we are hoping that an anthropic constraint can only be satisfied as a consequence of a symmetry. We will refer to such a situation as one in which we have an Anthropically Required Discrete Symmetry (ARDS). One might then hope that the symmetry could also explain the strong CP problem. We emphasize that in order to verify a claim like this, one would have to search through the $10^{100}$ states of the discretuum and prove that the overwhelming majority of states, which have light quark masses appropriate for anthropic needs, also have discrete symmetries which somehow solve the strong CP problem. We will shortly examine a toy model which suggests that the fraction of states which possess discrete symmetries is likely to be very small (so small quark masses, obtained randomly, might be much more likely). Still, while this possibility seems unlikely to us at the moment, its verification would be a triumph for the idea of a string landscape. This problem also exemplifies the kind of labor that would be involved 
in extracting predictions from the landscape.

The strong CP problem is our first serious obstacle to the anthropic selection mechanism. As we increase the energy scale, however, we encounter others. In particular, the masses of the $s, c$ and $b$ quarks, and their mixing angles, are all puzzling from this viewpoint. A similar statement applies to the $\mu$ and $\tau$ leptons (we will not attempt to deal with neutrino masses here). There is no obvious anthropic argument that these should be small. In the absence of some sort of approximate symmetry, we would expect them all to be of order the $W$ mass. We recall that typical explanations of these numbers rely on symmetries, but that typical flux vacua do not seem to have symmetries. We will comment on this issue further when we discuss proton decay in supersymmetric theories. There is also a wide range of similar problems associated with other rare processes involving violation of flavor symmetries of quarks and leptons. These are most severe if low energy SUSY, or some other criterion, forces us to consider new physics at scales that are not much higher than the weak scale.

There are further severe problems associated with proton decay. As we will explain, the flux discretuum is likely to predict low energy supersymmetry, so proton decay is potentially a very serious problem. Arguments based on Principle A, by themselves, provide at best a limit on the proton lifetime of order $10^{16}$ years or so[34]. So one cannot explain the large value of the proton lifetime from such considerations. Alternatively, one can, again, ask whether the proton lifetime can be accounted for by symmetries. But as for the question of light quark masses, the problem is that most states in the flux discretuum can be expected to possess no symmetries. Flux vacua that can account for observed phenomena have many small parameters (inverse fluxes), and typically have a rich hierarchy of mass scales, as we have indicated. There may be many processes which mediate proton decay at levels compatible with anthropic, but incompatible with experimental, bounds. In vacua with low energy SUSY this will certainly be a problem.

While in the absence of explicit examples, it is difficult to make a firm statement about the fraction of states which possess discrete symmetries, it is difficult to see how this fraction could be substantial. If one starts with some $F$-theory compactification with a large discrete symmetry, and a large number of possible fluxes (as required for a discretuum), one expects that the fluxes themselves will break the symmetries. The issues can be illustrated with a simpler and better understood example, that of the compactification of Type IIB theory on a $T_{6} / Z_{2}$ orientifold[36]. Here, at particular points in the moduli space, one has a large discrete symmetry, $Z_{2}^{5} \times S_{6}$. One can add fluxes, specified by twenty different integers, in such a way as 
to fix all of the complex structure moduli and the dilaton. Flux configurations break some or all of the discrete symmetries. Taking a value for just one of the integers reduces the symmetry to $S_{3} \times S_{3} \times Z_{2}^{4}$. Additional fluxes break further symmetry. In order to preserve a single $Z_{2}$, half of the 20 integers must vanish. By analogy, if there were, say, $10^{200}$ states, one might expect that only one in $10^{100}$ possess any discrete symmetry at all.

Of course, we might someday exhibit a large set of flux vacua for which there are discrete symmetries. Alternatively, if the low energy theory is not supersymmetric, the situation might be better. In such a case, we would have to invoke principle A selection arguments to account for the weak gauge hierarchy. Proton stability might then be understood if there were truly a desert up to the GUT scale. However, since no anthropic argument requires such a desert, and it is far from clear that typical flux vacua will produce one, it is not clear that we can understand the experimental bound on the proton lifetime in this way.

Indeed, the best way to solve most of the problems that we have enumerated is to imagine that anthropic arguments force us to a situation in which there is a desert up to the Planck or GUT scale, inhabited only by standard model particles. If one then assumed that the anthropic constraints on the light quark masses required us to have a set of discrete symmetries which solved the strong CP problem and explained the quark and lepton mass matrices, we would have a successful and predictive model of physics based on the discretuum, albeit a model that holds little excitement for experimentalists. At the moment, such a scenario seems unlikely to us, and the amount of theoretical labor involved in exploring it is daunting.

To summarize, many of the parameters of the standard model do not have an anthropic explanation, nor do they appear to be random numbers. Generic flux vacua do not support discrete symmetries which would provide a natural explanation for the values of these parameters. Barring remarkable accidents which will only be explained when we understand the detailed spectrum of flux vacua, it would seem that the generic prediction of models based on these meta-stable minima and principle $\mathrm{A}$, is in conflict with experiment.

\subsection{Might the Flux Discretuum Predict Low Energy Supersymmetry ?}

In the previous section, we have enumerated a number of phenomenological difficulties connected with the flux vacua. In this section, we will "suspend disbelief", supposing that some rational solution of these difficulties (symmetries which survive in a vast number of states, for example) will be found, and turn to the question: can the flux discretuum, coupled with anthropic 
arguments, lead to a prediction of low energy SUSY.

While the analysis of [7] is based on an action which is supersymmetric with small $\Lambda$, we have seen that in the vast majority of states, either one has unbroken supersymmetry, with a deep AdS minimum, or one has large supersymmetry breaking. Still, assuming a vast array of states, some number of states have small $W_{0}$ and small scale of supersymmetry breaking. In a fraction of these, as a result of random cancellations, the cosmological constant will be smaller than the scale expected from supersymmetry breaking. Indeed, we have seen that the probability of finding states with small $W_{0}$ goes as $W_{0} / M_{p}^{3}$.

The key to a prediction of low energy supersymmetry lies in the question of the cosmological constant. In non-supersymmetric states, we might expect that the probability of a low cosmological constant, of order $\delta^{4}$ (in Planck units), would be of order $\delta^{4}$. In supersymmetric states, the probability would be expected to be much higher. Roughly the probability of a negative cosmological constant of order $\delta^{4} \sim\left|W_{0}\right|^{2}$ would go as $\left|W_{0}\right|$ ! So low cosmological constant is far, far more probable in supersymmetric states. Now we need to fold this with the probability of breaking supersymmetry, with small cosmological constant. Suppose we take as our model for this phenomenon the possibility that low energy dynamics breaks supersymmetry. We might expect that this occurs in a significant fraction of states (for this purpose, note that a part in $10^{10}$ is a significant fraction). This supersymmetry breaking will generate a positive contribution to the cosmological constant ${ }^{19}$. We might expect, if the breaking is dynamical, that the distribution of this contribution is flat on a logarithmic scale. So, say, one in 50 of these states will have cosmological constant which is positive, and within an order of magnitude of $W_{0}$. So among the states with small cosmological constant, the vast majority will have some approximate supersymmetry. Our crude estimates suggest that the number of supersymmetric states with cosmological constant $\Lambda=10^{-120}$ is about 70 orders of magnitude larger than that of non-supersymmetric states with this small a value of $\Lambda$.

This is not actually what we want. It predicts that supersymmetry breaking is of order the scale set by the cosmological constant. However, principle A also requires us to look only at vacua with the right value of the weak scale. The usual SUSic solution of the gauge hierarchy problem implies that the number of approximately supersymmetric states, which have SUSY breaking scale of order the weak scale $(\sim 1 \mathrm{TeV})$, is much larger than the number of nonsupersymmetric states, which have the weak scale of this order.

\footnotetext{
${ }^{19}$ We emphasize that we are using conventional effective field theory thinking here. The actual situation in quantum gravity might be different. We are currently studying this puzzle.
} 
A rough estimate of the ratio of non-supersymmetric to supersymmetric states with suitable cosmological constant and weak scale might be $10^{-34} \times 10^{-120}=10^{-154}$. If we suppose, say, low scale supersymmetry breaking, with $F$ terms of order $10 \mathrm{TeV}$, than a plausible guess for the fraction of suitable supersymmetric states might be $10^{-10} \times 10^{-28} \times 10^{-2} \times 10^{-63}=10^{-103}$. The first factor is our crude guess as to the fraction of states with suitable supersymmetry breaking; the factor $10^{-28}$ accounts for the appropriate size of $W_{o}$; the factor of 100 accounts for the fraction which have suitable supersymmetry breaking scale; and the factor of $10^{-63}$ is the fraction of states for which accidental cancellations might be expected to give a suitably small cosmological constant. Note that, in this picture, the probability of states with suitable properties decreases rapidly with increasing susy breaking scale (roughly as the scale squared). Thus, the vast majority of states, which satisfy principle A for both the weak scale and the cosmological constant, have SUSY breaking of order the weak scale. Note again that in this more constrained ensemble, the preference, other things being equal, for small $W_{0}$ and the requirement of accidental cancellation of the remaining cosmological constant, implies that SUSY should be broken at low energies, as it is in gauge mediation.

These arguments suggest that the optimistic desert scenario described in the penultimate paragraph of the last section is not realized in the flux landscape. That is, we have identified a possible prediction which follows from the combination of the gross structure of the discretuum and the principle A. This prediction implies that we are saddled with all of the problems discussed in that section, such as the problem of proton decay and the smallness of the $\theta$ angle. One can only hope that the further requirement of small masses for the $u$ and $d$ quarks provide us with ARDS which solve all of these problems. Otherwise the anthropic predictions of the landscape will be in gross contradiction with experiment. On the positive side, note that, since all moduli are frozen at a rather high scale in the discretuum, one of the cosmological problems of gauge mediation is automatically solved.

It is worth noting, however, that the prediction of low energy supersymmetry breaking could well be an example of the sort of "rational explanation" we have discussed. It is unlikely that any anthropic condition would explain why the squark and slepton spectrum would exhibit the degree of degeneracy required by existing data. But if low energy supersymmetry breaking, with gauge interactions as the mediator, were generic, than at least this set of questions might find a rational explanation. 


\subsection{Above the TeV Scale: Cosmological Constraints}

For reasons which are ultimately tied to the renormalization group, anthropic arguments are sensitive to physics above the $\mathrm{TeV}$ scale primarily by virtue of its effect on cosmological history. Consequently, it is most useful to describe the constraints in terms of effective cosmological parameters, rather than Lagrangian parameters. We will find a reasonably wide range of cosmological parameters to be consistent with the constraints. A priori this suggests that an even wider variety of effective Lagrangians below the GUT scale will be consistent with them.

Assuming nuclear and stellar physics parameters to be fixed at their real world values ${ }^{20}$, the important cosmological parameters are the c.c., $\Lambda$, the dark matter density at the beginning of the matter dominated era, $\rho_{0}$, the normalization, $Q$, of the primordial density fluctuations, and the baryon asymmetry. Note that in making this statement we are committing ourselves to a rather particular model for structure formation, namely Cold Dark Matter with a HarrisonZeldovich spectrum of Gaussian primordial density fluctuations. We do this for simplicity only. If there exists a collection of flux vacua which predict the correct nuclear physics, and a distribution of galaxies generated by hot dark matter with cosmic string seeded density fluctuations, which are compatible with the existence of human life, then they should be included in the anthropically allowed list of flux vacua, even if they disagree violently with observation. In other words, our a priori restrictions can only enhance the possibility that anthropically chosen flux vacua could provide a predictive framework for fundamental physics.

Many authors have considered the possibility that anthropic considerations might explain the values of these parameters. Most analyses consider the effect of varying one parameter, while holding the others fixed. Even rather weak anthropic selection constraints are then quite impressive. Weinberg's constraint on the cosmological constant resulting from galaxy formation has the form:

$$
\Lambda \leq T Q^{3} \rho_{0}
$$

where $T$ is a number of order one. With other parameters fixed at their true values, this is close to the observed value. From the perspective of the discretuum, it requires that the discretuum have typical spacings in cosmological constant much smaller than $10^{-47} \mathrm{GeV}^{4}$. This is probably the most stringent constraint on the size of the discretuum. Even if the characteristic scale of the cosmological constant is as small as we might imagine - $(10 \mathrm{TeV})^{4}$, (as might be the case in

\footnotetext{
${ }^{20}$ In principle we should let both nuclear and cosmological parameters vary simultaneously to find the real anthropic range. This will imply an even wider scatter than we find in our current discussion.
} 
low energy gauge mediation) one still requires of order $10^{63}$ states, to address this issue alone. If anthropic considerations are relevant,this, as we have seen, is perhaps one of the stronger arguments for low energy gauge mediation.

One might hope to constrain the various parameters here independently. Rees and Tegmark[38] have attempted to provide independent anthropic arguments for the value of $Q$. They claim that it is bounded between $10^{-4}$ and $10^{-6}$, and suggest that Vilenkin's "principle of mediocrity" might show that most anthropically allowed solutions had $Q \sim 10^{-5}$ as implied by observation. However, Aguirre has argued that there are many regions of the cosmological parameter space which are compatible with even rather strong selection criteria; among these, the cosmological parameters can vary by many orders of magnitude[39] $]^{21}$. It is conceivable that stronger constraints, and more extensive simulations, will narrow the possibilities But it seems likely that they will not, and that typical states in the discretuum compatible with observers will predict cosmological parameters quite different than their observed values.

Within the framework of inflation and cold dark matter we have adopted, we can outline a number of potential problems with observational cosmology in the flux discretuum. In particular, we will now argue that $Q$ and $\rho_{0}$ are unlikely to be fixed to the same values in all anthropically allowed histories of the flux discretuum.

There are two leading particle physics candidates for dark matter, SUSY neutralinos, and axions. The discretuum freezes all moduli at a rather high energy scale, and thus does not provide us with any axion candidates. There are two problems with SUSic neutralinos, the first being that in the discretuum there is no reason to expect a conserved $R$ parity which makes the LSP stable. Let us lump this with the rest of the puzzles which we hope to solve with ARDS. We still have a problem, since we have claimed that the landscape predicts low energy SUSY breaking, which means that the LSP is a very light goldstino/gravitino, with rather strong (1-100 TeV scale) coupling to the rest of the contents of the universe. This is not a particularly good CDM candidate. It would appear that the generic prediction of the landscape is that dark matter is warm or hot, some combination of gravitini and neutrini. This is probably not consistent with observations.

We can instead proceed by assuming an as yet unidentified CDM candidate, which will be found to be predicted by the discretuum. By analogy with the situation for neutralinos, we anticipate that the physics determining its relic density will be affected by a large number of parameters in the TeV scale effective Lagrangian. As a result, its value can easily vary by

\footnotetext{
${ }^{21}$ We thank A. Aguirre and J. Primack for discussions of these and related issues.
} 
one or two orders of magnitude as we jump between different flux vacua with standard model parameters in their allowed ranges.

To discuss the likely values for $Q$ we must choose a model for the origin of fluctuations. For simplicity we have chosen inflation. This leads to a number of interesting questions about the discretuum. First, is inflation natural in this context? Second, is inflation anthropically favored? These are difficult questions, so we pose only tentative answers.

Typical models found in the inflation literature require fine tuning in the technical field theory sense. A class of models with minimal fine tuning is represented by lagrangians of the form

$$
\mathcal{L}=G_{i j}\left(\phi^{k} / m_{P}\right) \nabla \phi^{i} \nabla \phi^{j}-\frac{M^{6}}{m_{P}^{2}} V\left(\phi^{k} / m_{P}\right)
$$

where $M \sim 10^{16} \mathrm{GeV}$ and $m_{P}$ is the reduced Planck mass. Lagrangians of this type arise for bulk moduli in brane world models with SUSY broken on the branes, in which $M$ is the fundamental Planck scale and $m_{P}$ is a consequence of some modestly large extra dimensions $[42,43,44]$. They require $\mathcal{O}\left(1 / N_{e}\right)$ fine tuning, to explain $N_{e}$ e-foldings of inflation, and predict the right normalization for density fluctuations. We have seen that a large radius for the extra dimensions is a natural phenomenon in flux compactifications. It is also important that the semiclassical approximations for inflation and tunneling calculations are justified in these models by the small ratio $M / m_{P}$. The IIB solutions of the flux discretuum are indeed brane world scenarios of the above type, and so their moduli are appropriate inflaton candidates.

However, the interesting question is whether the number of e-foldings necessary to produce enough galaxies to guarantee the existence of human life is comparable to the number we need to explain observations. Our sense is that this is typically not the case. Thus, if say 30 e-foldings were enough to satisfy anthropic considerations, it would seem that there were many more histories of the flux universe compatible with life than were compatible with our observation of scale invariant correlations in the CMB over most of the horizon. We strongly suspect that the anthropically required number of e-foldings is quite a bit smaller than that required to fit the data. In the context of flux compactifications, and given the fine tuning necessary in order to get large $N_{e}$ one would suspect that most inflationary histories compatible with principle A have the minimum number of e-foldings.

Note that in the phrase "histories of the universe" , we include not only the different basins of attraction compatible with life, but also the initial conditions for the inflationary trajectory which comes to rest in a given basin. The possibility of tunneling between different meta-stable 
dS minima ensures that there will be many different trajectories which access a given basin of attraction. Each will give a different number of e-foldings of inflation and a different value of $Q$. The values of $Q$ may differ by as much as an order of magnitude or so.

Rees and Tegmark's bounds on $Q$ do not appear to be independent of $\Lambda$ and are in fact evaluated only for $\Lambda=0$. Thus, for example, the upper bound comes from the statement that dense supermassive galaxies would form, in which collisions would disrupt planetary orbits on a time-scale too short for our kind of life to evolve. However, if $\Lambda$ were larger, then the value of $Q$ at which this occurs would also be larger, so although this bound may have a somewhat different functional form than Weinberg's, it has a similar correlation between large values of $Q$ and $\Lambda$. Consequently, a uniform distribution in the anthropically allowed region of these two bounds, would favor large values of $Q, \rho_{0}$ and $\Lambda$, constrained only by the underlying theory (the discretuum in our case). Given the fact that Weinberg's bound already overshoots the observed value for $\Lambda$, when $Q$ and $\rho_{0}$ are given their observed values, we doubt that even the principle of mediocrity can rescue the situation. Rather, the anthropic prediction for the three parameters will simply disagree with observation without further theoretical input. We have argued above that the discretuum scenario is unlikely to supply that extra input. Of course, there is always the possibility that further explorations of the discretuum will somehow constrain the relative values of these parameters, or that stronger anthropic arguments will fix them to their observed values. But both possibilities seem unlikely.

\section{Conclusions}

Our remarks have ranged over a wide range of issues. Let us try to put some order to our observations. We have argued that the concept of effective potential has no exact meaning in theories of quantum gravity. We believe that it is therefore not a coincidence that one cannot propose a systematic method of improving the calculations which suggest the existence of a discretuum in string theory. For the case of the AdS discretuum, a possible hypothesis is that it corresponds to a large class of three dimensional CFT's and that the effective potential computation is some sort of approximation to the problem of finding RG fixed points. Each point in this discretuum would be an isolated, self consistent model of quantum gravity in AdS space.

For meta-stable dS minima, we must solve two problems. We must show that the disastrous Big Crunch instability is harmless. If there are non-vanishing tunneling amplitudes between 
any two dS minima, even one non-vanishing amplitude to tunnel to the Big Crunch might pose a problem. To resolve it, one would either have to find a quantum resolution of the Big Crunch, or prove Susskind's conjecture that the singularities are no more dangerous than those of black holes in asymptotically flat space-time.

Even if this problem is resolved, we must find the quantum theory of the Big Bang cosmologies in the Dine-Seiberg region, which are the putative stable endpoint of meta-stable dS decay. And we must show that the quantum observables of this model of gravity indeed contain information relevant to a hypothetical observer during a particular dS era. The question of whether these minima of the effective potential are relevant to real models of quantum gravity is not yet answered.

Leaving aside these questions of principle, we investigated the likelihood that the discretuum could lead to correct postdictions for existing experimental data, or predictions for experiments not yet done. We argued that a great deal of a priori data will have to be supplied, and followed the suggestion of Susskind that the principle A might be the most economical way to package this additional input. We argued that the renormalization group suggests a problem with this approach.

In order to correctly select the standard model gauge group, we will have to show that the discretuum does not contain many models which reproduce nuclear physics as we know it, but disagree with experiment at the few $100 \mathrm{GeV}$ energy scale. We argued that $S U(3)$ may be selected as the group of the strong interactions by detailed interplay between nuclear physics and stellar structure, but many possible weak interaction theories are likely compatible even with the principle A.

Assuming an argument which fixes the low energy gauge group to be the standard model, we found a large number of residual problems. These included the existence of precisely two heavier generations of quarks and leptons, and the many parameters in the standard model which take on values which are not determined anthropically, but are far from random. This problem was exacerbated by our argument that low energy SUSY probably is a prediction of the discretuum. The combined anthropic constraints on the cosmological constant and the weak scale, and the general structure of the discretuum, favor SUSY breaking at about the weak energy scale. The result is that our low energy models have a large number of parameters that are severely constrained by experiment, but not nearly so severely by anthropic arguments.

We suggested one possible resolution of this problem. Anthropic arguments favor very 
light $u$ and $d$ quarks, as well as a small electron mass. One might imagine showing that within the discretuum, these values could be obtained in an overwhelming more probable way, only in the presence of a large set of discrete symmetries. One then hopes that these symmetries will be enough to explain the otherwise peculiar values of a host of parameters in the low energy Lagrangian. We do not see any indication that this optimistic hypothesis is true. Furthermore, just like the problem of predicting the standard model gauge group, verifying the hypothesis involves a herculean task. One must survey the entire set of metastable dS minima with the right values of the cosmological constant, the weak scale, and the first generation fermion masses and show that the overwhelming majority of them also have the correct order of magnitude predictions for the all of the "peculiar" parameters in the supersymmetric standard model.

Our final topic was an analysis of strong selection constraints on physics above the scale of the standard model. These constraints operate through cosmology, so we presented our analysis in terms of effective cosmological parameters, rather than the high energy Lagrangian parameters which might determine them. The center of our analysis was the anthropic constraint on the cosmological constant, which is really a relation between that parameter, the ratio of dark matter to photons, and the amplitude of primordial fluctuations. We argued that all of these were expected to fluctuate as we move around the discretuum.

Our first result was a negative one: the combination of the freezing of moduli in the discretuum, and our prediction of low energy SUSY, left us with no apparent candidates for cold dark matter. The discretuum, plus the constraints on physics at the standard model scale, seems to predict a hot dark matter universe, which is allowed anthropically, but not observationally. Assuming that, once we had better knowledge of the discretuum, a CDM candidate would be found in the majority of anthropically allowed states, we argued that the Weinberg relation between $\Lambda, \rho_{0}$, and $Q$, was likely to predict expectation values for these parameters in the anthropically allowed ensemble of flux vacua, which were inconsistent with observations.

Finally, we noted that, since models with $N_{e}$ e-foldings of inflation require fine tunings which are at least of order $1 / N_{e}$, anthropic arguments suggest that the discretuum will predict a large probability for models with the smallest number of e-foldings compatible with life. If that number is of order 10, our own universe might appear somewhat special. However, this is much less of a problem than most of the erroneous predictions of the anthropically constrained landscape.

Certainly, if there are any points in the landscape at all, there are many more than are 
accessible to semiclassical analysis. Furthermore, if one wants to make a real assault on the problem of making connections between the discretuum and experiment, one must find ways to calculate the cosmological constant in any given vacuum with precision $10^{-120}$. Thus, if one wants to put the lie to the dismal picture of discretuum phenomenology that we have painted, one must first address the question of the precise mathematical formalism to which the effective potential is an approximation. The proposal of Silverstein[10] is a step in this direction for AdS points in the discretuum. However, we believe we have made it clear that this is a very different

problem than finding the mathematical framework for meta-stable dS minima. At present we have no clue about how one might go about computing corrections to the classical predictions for these states, in a systematic manner. Thus, it appears to us that the attempt to address the phenomenology of the discretuum at a level deeper than our own analysis, must first face up to the problems of principle with which we began our discussion.

Acknowledgements: We thank A. Aguirre, M. Douglas, S. Kachru, J.Primack, E. Silverstein, L. Susskind, S. Thomas, S. Trivedi and J. Wells for discussions. Conversations with A. Aguirre and Willy Fischler were particularly important in shaping our thinking on many of these issues. We appreciate detailed comments from A. Aguirre, S. Kachru, J. Polchinski and L. Susskind on an early version of the manuscript.

This work supported in part by the U.S. Department of Energy.

\section{References}

[1] R. Bousso and J. Polchinski, JHEP 0006, 006 (2000) [arXiv:hep-th/0004134].

[2] J. L. Feng, J. March-Russell, S. Sethi and F. Wilczek, Nucl. Phys. B 602, 307 (2001) [arXiv:hep-th/0005276].

[3] K. Becker and M. Becker, Nucl. Phys. B 477, 155 (1996) [arXiv:hep-th/9605053].

[4] J. de Boer, R. Dijkgraaf, K. Hori, A. Keurentjes, J. Morgan, D. R. Morrison and S. Sethi, Adv. Theor. Math. Phys. 4, 995 (2002) [arXiv:hep-th/0103170].

[5] S. B. Giddings, S. Kachru and J. Polchinski, Phys. Rev. D 66, 106006 (2002) [arXiv:hepth/0105097].

[6] B. S. Acharya, arXiv:hep-th/0212294 and arXiv:hep-th/0303234.

[7] S. Kachru, R. Kallosh, A. Linde and S. P. Trivedi, arXiv:hep-th/0301240.

[8] L. Susskind, arXiv:hep-th/0302219. 
[9] M. R. Douglas, JHEP 0305, 046 (2003) [arXiv:hep-th/0303194]; S. Ashok and M. R. Douglas, arXiv:hep-th/0307049.

[10] E. Silverstein, Talk at Strings 2003, Kyoto Japan, July 2003.

[11] T. Banks, arXiv:hep-th/0007146.

[12] Inflationary cosmology provides the only setting for a potentially predictive (and hense scientific) discussion of the anthropic principle. To our knowledge, this point was first made by A.D. Linde in A.D. Linde, Nonsingular Regenerating Inflationary Universe Print-820554 (Cambridge); The New Inflationary Universe Scenario in The Very Early Universe, eds. G.W. Gibbons, S. W. Hawking and S. Siklos (Cambridge University Press, Cambridge 1983) 205-249. It is also discussed in A.D. Linde, Partile Physics and Inflationary Cosmology, (Chur, Switzerland, Harwood, 1990)

[13] See M. Fabinger and E. Silverstein, arXiv:hep-th/0304220, and references therein; see also P. Kraus, F. Larsen and S. P. Trivedi, JHEP 9903, 003 (1999) [arXiv:hep-th/9811120].

[14] W. Fischler and L. Susskind, Phys. Lett. B 171, 383 (1986); W. Fischler and L. Susskind, Phys. Lett. B 173, 262 (1986).

[15] T. Banks, arXiv:hep-th/0306074.

[16] T. Banks, arXiv:hep-th/0011255.

[17] T. Banks and M. Dine, JHEP 0110, 012 (2001) [arXiv:hep-th/0106276].

[18] S. Gukov, C. Vafa and E. Witten, Nucl. Phys. B 584, 69 (2000) [Erratum-ibid. B 608, 477 (2001)] [arXiv:hep-th/9906070].

[19] T. Banks, M. Dine and E. Gorbatov, in preparation.

[20] T. Banks, arXiv:hep-th/0211160.

[21] E. Farhi and A. H. Guth, Phys. Lett. B 183, 149 (1987); E. Farhi, A. H. Guth and J. Guven, Nucl. Phys. B 339, 417 (1990).

[22] E. Witten, arXiv:hep-th/0106109.

[23] T. Banks and W. Fischler, arXiv:hep-th/0102077.

[24] L. Susskind, arXiv:hep-th/0302219.

[25] T. Banks, M. Dine and N. Seiberg, Phys. Lett. B 273, 105 (1991) [arXiv:hep-th/9109040].

[26] T. Banks, M. Dine and L. Motl, JHEP 0101, 031 (2001) [arXiv:hep-th/0007206].

[27] T. Banks and M. Dine, Phys. Rev. D 53, 5790 (1996) [arXiv:hep-th/9508071].

[28] T. Banks, Phys. Rev. Lett. 52, 1461 (1984). 
[29] A.D. Linde, Inflation And Quantum Cosmology, Print-86-0888 (June 1986), in: Three hundred years of gravitation, (Eds.: Hawking, S.W. and Israel, W., Cambridge Univ. Press, 1987), 604-630.

[30] J. Garriga and A. Vilenkin, Phys. Rev. D 61, 083502 (2000) [arXiv:astro-ph/9908115].

[31] S. Weinberg, arXiv:astro-ph/0005265.

[32] T. Banks, M. Dine, P. J. Fox and E. Gorbatov, arXiv:hep-th/0303252.

[33] T. Banks, M. Dine and M. Graesser, arXiv:hep-ph/0210256.

[34] Weinberg[35] attributes this observation to M. Goldhaber.

[35] S. Weinberg, Rev. Mod. Phys. 61, 1 (1989).

[36] S. Kachru, M. B. Schulz and S. Trivedi, arXiv:hep-th/0201028.

[37] M.B. Green, J. Schwarz and E. Witten, Superstring Theory, Cambridge University Press, Cambridge 1987.

[38] M. Tegmark and M. J. Rees, Astrophys. J. 499, 526 (1998) [arXiv:astro-ph/9709058].

[39] A. Aguirre, Phys. Rev. D 64, 083508 (2001) [arXiv:astro-ph/0106143].

[40] S. Dimopoulos and S. Thomas, private communication and in preparation.

[41] A. E. Nelson, Phys. Lett. B 143, 165 (1984); S. M. Barr, Phys. Rev. Lett. 53, 329 (1984).

[42] P. Horava and E. Witten, Nucl. Phys. B 460, 506 (1996) [arXiv:hep-th/9510209]; E. Witten, Nucl. Phys. B 471, 135 (1996) [arXiv:hep-th/9602070].

[43] T. Banks and M. Dine, Nucl. Phys. B 479, 173 (1996) [arXiv:hep-th/9605136].

[44] T. Banks, arXiv:hep-th/9906126. 